\title{
10 años de Tennis Play \& Stay: Una reseña sobre la comunicación y la enseñanza del tenis en Alemania, desde (pre)escolar hasta las edades más avanzadas
}

\author{
Philipp Born, Hans-Peter Born, Rüdiger Bornemann, Alexander Jakubec y Tobias Vogt \\ Universidad Alemana del Deporte de Colonia, Alemania.
}

\section{RESUMEN}

Alemania cuenta con una larga tradición en la formación de entrenadores muy capacitados, en la que siempre ha utilizado enfoques metodológicos para el aprendizaje y para la enseñanza del tenis. La implementación de la campaña Play \& Stay de la ITF en 2007 impulsó especialmente los programas existentes aportando nuevas ideas y un sistema de nivel superior para el tenis alemán. A pesar de algunas dificultades menores, la campaña $P \& S$ fue y es muy exitosa en Alemania

\author{
Palabras clave: Play \& Stay, \\ Alemania, campañas, \\ programas e iniciativas \\ Recibido: 10 Feb 2017 \\ Aceptado: 08 Abr 2017 \\ Autor correspondiente: \\ Philipp Born, Universidad \\ Alemana del Deporte de Colonia, \\ Alemania \\ Correo electrónico: \\ p.born@dshs-koeln.de
}

\section{INTRODUCCIÓN}

Alemania ha utilizado siempre enfoques metodológicos para el aprendizaje y para la enseñanza del tenis. Además, tiene una larga tradición en formación de entrenadores bien capacitados. La implementación de la campaña Play \& Stay por parte de la ITF en 2007 proporcionó un impulso especial a los programas existentes.

\section{HISTORIA}

Desde 1970, la Federación Alemania de Tenis (DTB) ha iniciado varios programas de tenis y ha avanzado con la cooperación entre las escuelas y los clubes. Además, Schönborn y cols. (1970) introdujeron los primeros enfoques metodológicos para la enseñanza del tenis, recomendando que los niños comiencen con pequeñas tablas de madera, algo llamado 'Holzbrett-Tennis'. Basándose en estas ideas, la comisión de escuelas de tenis publicó una serie de textos ('Lehrpläne des DTB' u.a. Brinker, W. y Dreibholz, K., 1975), incluyendo, fundamentalmente, instrucciones orientadas hacia la técnica. Bornemann (1977) publicó la primera teoría orientada al juego, sin embargo, en ese momento, solamente unos pocos entrenadores, principalmente provenientes de las universidades, aplicaron este enfoque orientado al juego.

Subsiguientemente, desde los años 1980 ha habido varias iniciativas y programas relacionados con el tenis infantil, con el material adaptado ('ST-Ball') y formas de aprender y enseñar el tenis de manera efectiva ('Kleinfeldtennis'). La asociación regional de Württemberg fue la primera en comenzar con la iniciativa de seleccionar niños talentosos para jugar al tenis a fines de los 80 . Siguiendo con esta iniciativa, se organizaron los primeros torneos regionales en canchas más pequeñas utilizando materiales adaptados y juegos con habilidades motrices que continúan hoy en día.

Comenzaron también iniciativas como "DTB-Talentcup", un equipo de competición para los mejores jugadores menores de 11 años y "Tenis en la calle", una iniciativa anterior a la implementación de la campaña Play \& Stay en 2007, que aún continúa. Sin embargo, todos estos programas e iniciativas eran mayormente regionales y no proponían cambios en las reglas de tenis para las competiciones infantiles. 


\section{LA IMPLEMENTACIÓN DE PLAY \& STAY}

Además de los programas existentes, la campaña Play \& Stay introdujo en Alemania nuevas ideas y un sistema de métodos de entrenamiento holístico, con canchas y tamaños de raquetas

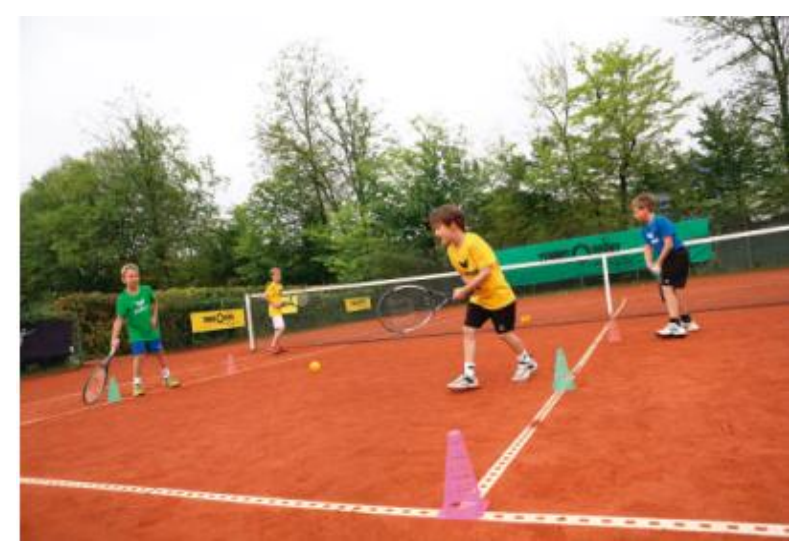

diferentes, otros tipos de pelotas y torneos. Después del inicio de la campaña en 2007, la DTB y sus asociaciones regionales implementaron Play \& Stay en la formación de programas de formación para sus entrenadores de todos los niveles, además del "Campus en línea", la plataforma de entrenamiento oficial en línea de la DTB. Con todo esto, el enfoque orientado al juego recibió una nueva proyección a la hora de introducir el tenis. Hoy, Play \& Stay es el concepto básico y el programa para desarrollo de talentos utilizado por la mayoría de los clubes de tenis alemanes para conseguir nuevos miembros. La mayoría de los iniciantes comienzan con Tennis 10s, Cardio Tennis, Tennis Xpress u otros programas relacionados con Play \& Stay. En particular la etapa 1 de pelota verde ha logrado gran aceptación entre los iniciantes adultos y adolescentes.

\section{APLICACIONES PRÁCTICAS}

\section{CAMPAÑAS E INICIATIVAS}

Las iniciativas Play \& Stay, 'Tennis $10 \mathrm{~s}^{\prime} \mathrm{y}$ 'Cardio Tennis' forman parte del tenis alemán desde 2007. En 2014 se tradujo el manual de Tennis Xpress al Alemán y desde entonces forma parte de la formación de los entrenadores.

Además de estos programas, la DTB, en particular su grupo de trabajo sobre Play \& Stay, y sus asociaciones regionales han comenzado varias iniciativas y programas en base a dicha campaña.

\section{Congreso Play \& Stay 2010}

El primer congreso de Play \& Stay se llevó a cabo en Leipzig, en 2010, para fomentar una mayor difusión de la campaña en Alemania. Su título fue 'Spielend in die Zukunft', que tiene el doble significado: "sin esfuerzo hacia el futuro" y "jugar en el futuro". El congreso estaba dirigido a todos los entrenadores de tenis, representantes interesados de las asociaciones regionales y clubes de tenis, lo mismo que a los maestros que introducen el tenis en los colegios.

\section{Competición}

Tradicionalmente, el tenis alemán se basa en y está impulsado por los clubes de tenis y las competiciones por equipos de los clubes, organizados por las asociaciones regionales. En éstos, los equipos de los clubes juegan entre sí, en un sistema de ligas. Los equipos constan de seis (o cuatro jugadores), que juegan seis (o cuatro) individuales y tres (o dos) dobles. Antes de la campaña Play \& Stay estos partidos por equipos de clubes se jugaban en grupos de todas las edades, desde niños hasta seniors. La campaña Play \& Stay agregó competiciones también para las canchas rojas, naranja y verdes. Estos partidos se juegan en equipos de niños / niñas solamente, o en equipos mixtos. Además de los partidos de individuales y de dobles, los juegos con diferentes habilidades motrices son parte de la competición (obligatorio en algunas regiones).

Además de los partidos por equipos en los clubes, la cantidad de competiciones aumentó significativamente en Alemania después de la implementación de la campaña Play \& Stay en 2007. No solamente hay más torneos regulares de individuales para niños menores de 10 años, también hay series de torneos regionales para jugadores de cancha roja, naranja y verde, que concluyen en un torneo regional de Maestros al final de cada temporada de verano. Durante dos años, también se llevó a cabo un torneo nacional de Maestros, que fue suprimido pues muchas asociaciones regionales no querían campeonatos nacionales para esas edades. Uno de los lemas de Play \& Stay, "la competición impulsa al deporte" fue también un elemento decisivo para establecer la idea de Play \& Stay en Alemania.

Número Internacional de Tenis (ITN) = 'Leistungsklassen'

Basándose en la idea del ITN, un sistema de clasificación para jugadores de todos los niveles de rendimiento, la DTB desarrolló un sistema de clasificación llamado 'Leistungsklassen' (LK) que puede traducirse como clases para

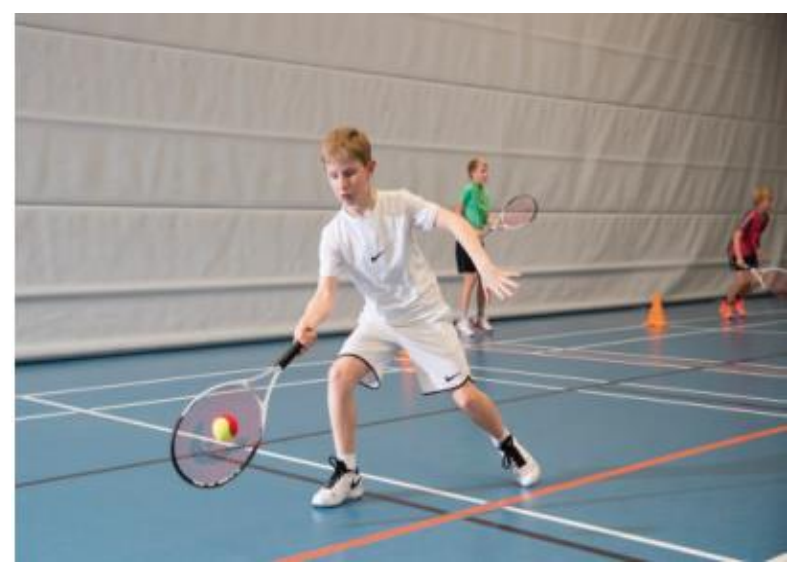

el alto rendimiento. Además de la clasificación alemana existente que sólo es significativa para los jugadores 
avanzados y de alto rendimiento en Alemania, la LK dió un número a cada jugador que estuviera compitiendo en un equipo de un club, o en torneos, o en ambos. Comenzando con LK 1 (todos los jugadores con clasificación nacional alemana) hasta LK 23, todos los jugadores fueron clasificados. Al ganar a un jugador clasificado en un nivel igual o superior, se puede mejorar el LK .El sistema ayudaba a aumentar el número de competiciones y de jugadores compitiendo, de todas las edades y de todos los niveles, particularmente en el tenis aficionado ('Breitensport').

\section{"Distintivo de tenis" ('Tennis Sportabzeichen')}

Si bien existe desde 1988, la DTB relanzó el "distintivo de tenis" en 2016 como parte de la campaña Play \& Stay en Alemania. En base al concepto Play \& Stay, el "distintivo de tenis" es una prueba de tenis específica en la que pueden participar los jugadores de todas las edades y niveles para ver su rendimiento en diferentes tareas del juego de tenis. La prueba está diseñada para las cuatro etapas del Play \& Stay (roja, naranja, verde, amarilla) y la exitosa terminación se recompensa con certificados y/ o medallas bronce, plata y oro. La prueba contiene cuatro tareas específicas para el tenis que cubren la parte técnica, táctica, de coordinación, y física del juego de tenis. Las diferentes tareas se recompensan con puntos, que sumados, determinan el nivel y certificación alcanzados.

\section{"Alemania juega al tenis" ('Deutschland spielt Tennis')}

Desde 2007, los clubes de tenis alemanes comienzan cada temporada de verano con un evento anual nacional llamado "Alemania juega al tenis". Durante este día se celebran varios eventos en clubes de tenis, para iniciar la temporada veraniega, promocionar el tenis en Alemania y atraer a más gente nueva para jugar al tenis.

\section{Talentinos}

"Talentinos" es un concepto de entrenamiento y marketing basado en la campaña Play \& Stay. El concepto incluye la llamada "escuela de pelota" para niños de 4 a 6 años de edad. Esta "escuela de pelota" se divide en 3 etapas, según la idea de "entrenamiento básico" ('Grundlagentraining') y como elemento básico para el desarrollo de talentos del tenis alemán, buscando una mayor adquisición de habilidades motrices antes de la temprana especialización en el tenis. Después de la "escuela de pelota" el concepto de "Talentinos" continúa con las 3 etapas de Play \& Stay, rojo, naranja y verde.

\section{DESAFÍOS}

La DTB se divide en 18 asociaciones regionales que están a cargo del desarrollo del tenis en la región. Esta es la razón principal por la cual hay algunos enfoques diferentes dentro de Alemania con respecto a Play \& Stay. Un ejemplo es el ancho de la cancha naranja. La mayoría de las asociaciones regionales juegan sus competiciones de cancha naranja en $18 \mathrm{~m} . \times 8.23 \mathrm{~m}$., el ancho de una cancha estándar). Pero hay regiones que juegan en una cancha de $18 \mathrm{~m}$. x 6.40m. Esto puede confundir a jugadores que compiten en diferentes regiones.

Si bien la idea de Play \& Stay y sus programas e iniciativas se promueven en toda Alemania, y forman parte de todos los programas de formación de entrenadores, aún hay algunos entrenadores que no utilizan el material adaptado y no siguen las recomendaciones. La razón principal son los costos y los esfuerzos adicionales que implica la adopción del Play \& Stay

\section{CONCLUSIÓN}

La implementación de la campaña Play \& Stay por parte de la ITF en 2007 proporcionó nuevas ideas y un sistema de nivel superior para el tenis alemán. Teniendo una larga y exitosa historia de programas de participación en el tenis, la DTB incluyó sus programas existentes en la campaña Play \& Stay, comenzando con programas nuevos e innovadores para atraer a más gente a jugar al tenis. A pesar de algunas dificultades menores, la campaña P\&S fue y es muy exitosa en Alemania.

\section{REFERENCIAS}

Bornemann, R. (1977). Tennistechnik ein dynamischer Stereotyp?. Tennis in Schulen und Hochschulen, Bd.1, 46-62.

Brinker, W. \& Dreibholz, K. (1975). Tennis-Lehrplan, 1: Holzbrett Tennis. München: BLV.

Die Talentinos (n.d.). Retrieved June 30, 2017, from Kinder Tennis: https://kinder.tennis.de/konzept/die-talentinos

Initiativen und Projekte (n.d.).Retrieved June 30, 2017, from DTBTennis: http://www.dtb-tennis.de/Initiativen-undProjekte/

Leistungsklassen (n.d.). Retrieved June 30, 2017, from DTBTennis: http://www.dtb-tennis.de/TennisNational/Leistungsklassen

Schönborn, R., Biesenkamp, G., Bolardt, A. \& Brabenec, J. (1970 Kindertennis. Hannover: Deutscher Tennis Bund.

Street Tennis Tour (n.d.). Retrieved June 30, 2017, from WTV: http:// www.wtv.de/street-tennis-tour 
CONTENIDO ITF ACADEMY RECOMENDADO (HAZ CLICK ABAJO)

\section{TTF Academy}

Derechos de Autor (c) 2017 Philipp Born, Hans-Peter Born, Rüdiger Bornemann,

Alexander Jakubec et Tobias Vogt



Este texto está protegido por una licencia CreativeCommons 4.0.

Usted es libre para Compartir -copiar y redistribuir el material en cualquier medio o formato- y Adaptar el documento -remezclar, transformar y crear a partir del material - para cualquier propósito, , incluso para fines comerciales, siempre que cumpla la condición de:

Atribución: Usted debe dar crédito a la obra original de manera adecuada, proporcionar un enlace a la licencia, e indicar si se han realizado cambios. Puede hacerlo en cualquier forma razonable, pero no de forma tal que sugiera que tiene el apoyo del licenciante o lo recibe por el uso que hace de la obra.

Resumendelicencia - Textocompletodelalicencia 